\title{
PREVALENCIA DE HIDATIDOSIS HUMANA MEDIANTE TÉCNICAS DE IMAGEN EN YANAHUANCA, PASCO
}

\author{
Prevalence of Human Echinococcosis Using Imaging Techniques in \\ Yanahuanca, Pasco
}
Elizabeth Chumbe G. ${ }^{1}$, Luis Lopera B. ${ }^{1}$, Eduardo Barrón G. ${ }^{1}$, Berenice Ninaquispe B. ${ }^{1}$, César Gavidia Ch. ${ }^{1,2}$

\section{RESUMEN}

El objetivo del estudio fue determinar la prevalencia de hidatidosis humana en nueve comunidades rurales del distrito de Yanahuanca, Pasco, empleando como técnicas de diagnóstico la ecografía abdominal y la radiografía de tórax. Además, se evaluó la asociación entre las variables edad, género y comunidad de procedencia y la presencia de pobladores positivos a quistes hidatídicos. La ecografía se realizó a 949 personas (52\% de la población mayor de 5 años) y el examen radiográfico a 829 (45.6\%). La prevalencia general de hidatidosis fue de 5.5\% (52/949) con un intervalo de confianza al 95\% de $4.1 \mathrm{a}$ 7.1\%. Se halló asociación estadística $(\mathrm{p}<0.05)$ entre edad con el resultado positivo al examen ecográfico, siendo las personas mayores de 40 años las que presentaron un mayor porcentaje. La relación de quistes hidatídicos hepáticos / pulmonares fue de 5.1. El $56 \%$ (25/45) de los quistes detectados por ecografía presentaron parcial o completa calcificación. La prevalencia obtenida es una de las más altas en Latinoamérica, posiblemente por la convivencia del poblador andino con el perro, dado su uso en el cuidado y pastoreo del ganado ovino, además de las deficientes condiciones higiénico-sanitarias, y bajos niveles socioeconómicos y culturales.

Palabras clave: humano, prevalencia, quiste hidatídico

\section{ABSTRACT}

The objective of this study was to determine the prevalence of human echinococcosis in nine communities of the district of Yanahuanca, Pasco, through abdominal echography and thorax radiography. In addition, the association between age, gender, and community with the disease was evaluated. Echographic examination was conducted in 949 persons ( $52 \%$ of the population over 5 years of age) and X ray test was performed in 829 (45.6\%). The overall prevalence of human cystic echinococcosis was $5.5 \%$ (52/949) and the 95\% confidence interval varied from 4.1 to $7.1 \%$. There was statistical association $(\mathrm{p}<0.05)$ between age with positive results when using the ultrasound exam. The liver / lung hydatid cyst ratio was 5:1. Partial or total calcified cysts were detected by

\footnotetext{
${ }^{1}$ Laboratorio de Medicina Veterinaria Preventiva, Facultad de Medicina Veterinaria, Universidad Nacional Mayor de San Marcos, Lima

2E-mail: cgavidiac@unmsm.edu.pe,cmgavidia@yahoo.com
} 
echographical examination (56\%, 25/45). The prevalence of human hydatidosis in this endemic area of Peru is one of the highest in Latin America, possibly due to the close interaction with dogs, as they shepherd the flocks, plus poor sanitation and hygiene conditions in the area.

Key words: human, prevalence, hydatid cyst

\section{INTRODUCCIÓN}

La hidatidosis humana es una zoonosis parasitaria, reconocida como un problema de salud pública importante en regiones de crianza ovina en el mundo. El hombre es considerado como un hospedador intermediario accidental tras la ingestión de huevos de Echinococcus granulosus por el contacto con perros parasitados (Larrieu et al., 2004).

En América del Sur, los países considerados altamente endémicos son Argentina, Brasil (Estado de Rio Grande do Sul), Chile, Perú y Uruguay (Eckert y Desplazes, 2004; Larrieu et al., 2004). En el Perú, los focos hidatídicos de gran importancia se encuentran en la Sierra Central (Junín, Pasco y provincias de Lima) y en el sur (Cusco y Puno), las cuales reúnen las características ecológicas, culturales, económicas y sociales que permiten el mantenimiento del ciclo biológico del metacéstodo (quiste hidatídico) (Chuquisana et al., 2000).

En la sierra central, principalmente en las provincias de Lima y Junín, las prevalencias reportadas para hidatidosis humana, determinadas a través de ecografía abdominal y radiografía de tórax, son altas (4.3 a 9.1\%) (Moro et al., 1999, 2005); sin embargo, los datos proporcionados sobre las prevalencias de esta enfermedad en la zona de Pasco son limitadas, pues solo se basan en registros hospitalarios, los cuales no reflejan la verdadera problemática de esta zoonosis. La importancia sanitaria de esta enfermedad reside en la alta morbilidad que produce, además de ocasionar pérdidas económicas debido a gastos por cirugía, medicamentos, estancia hospitala- ria, días no trabajados, consecuencias emocionales y sociales y, en algunos casos, por la muerte de las personas afectadas (Náquira et al, 1989; Aliaga y Oberg, 2000).

En este contexto, es importante conocer la situación epidemiológica de un área potencialmente endémica, utilizando métodos de diagnóstico adaptados a condiciones de campo, de bajo costo operativo, de alta sensibilidad y especificad como las técnicas de imagen (ecografía y radiografía). Este estudio tuvo como objetivo estimar la prevalencia de hidatidosis humana en zonas rurales del distrito de Yanahuanca, Pasco, utilizando las técnicas de imagen como métodos de investigación para detectar quistes hidatídicos hepáticos y pulmonares.

\section{Materiales y MÉTOdos}

El estudio se llevó a cabo en agosto de 2004 en nueve comunidades del distrito de Yanahuanca, provincia Daniel Alcides Carrión, departamento de Pasco. Las comunidades fueron Andachaca, Astobamba, Ayayog, Huarautambo, Tambochaca, Tambopampa, Santiago Pampa, Uchumarca y 12 de Octubre, localizadas entre los 3200 a $4500 \mathrm{msnm}$. Entre sus principales actividades económicas destaca la ganadería, predominando la crianza de ovinos, y donde el beneficio del ganado es casero y los perros se emplean rutinariamente para el cuidado y pastoreo de las ovejas.

Se realizó un censo en las nueve comunidades para determinar el tamaño de la población y sus características demográficas. 
Luego se calculó el tamaño muestreal en base a la fórmula para estimación de proporciones con población conocida. Se tomó como referencia la prevalencia de 5.7\% reportada para la SAIS Túpac Amaru (Moro et al., 1997). El nivel de confianza empleado fue del $95 \%$ con un error de $1.25 \%$, resultando en un tamaño muestral estimado de 818 personas.

En las personas participantes se realizó un examen clínico general a cargo de un equipo médico. Los niños menores de cinco años y las mujeres que dieron positivo a la prueba de embarazo en orina fueron excluidas de participar en el estudio como medida de prevención. Se evaluó a las personas seleccionadas mediante exámenes ecográficos y radiológicos, previo consentimiento en el Centro de Salud de cada comunidad.

La ecografía hepática se realizó en posición supina, utilizando un ecógrafo portátil Shimatzu (Shimazonit SDL-32, 3.5 MHZ), con parafina líquida como medio de transducción. Los quistes fueron clasificados según el esquema de la Organización Mundial de la Salud (WHO, 2003):

Tipo CL: Lesión quística unilocular con contenido anecoico uniforme (pared no visible). No son signos patognomónicos.

Tipo CE1: Quiste unilocular con contenido anecoico uniforme y con signos patognomónicos que incluye pared quística visible y signo de nevado.

Tipo CE2: Quiste multivesicular, multiseptado.

Tipo CE3: Contenido anecoico con desprendimiento de la membrana que puede aparecer flotando.

Tipo CE4: Contenido degenerativo, hipoecoico o hiperecoico, heterogéneo, no presentan vesículas hijas.

Tipo CE5: Quistes caracterizados por presentar paredes calcificadas bien definidas con forma de arco, produciendo un cono de sombra. El grado de calcificación puede variar de parcial a completo.
Las radiografías de tórax se tomaron en posición posterior-anterior, inmediatamente después del examen ecográfico. Las placas fueron diagnosticadas por un radiólogo que desconocía los resultados del ultrasonido. Los quistes hidatídicos pulmonares fueron diagnosticados utilizando los criterios de Beggs (1985): imágenes radiográficas redondeadas y de bordes definidos, cerrados y localizados centralmente.

Se consideró como positivo, para los fines de determinar la prevalencia general de hidatidosis, a aquellas personas con al menos un examen positivo de las dos pruebas realizadas. Se determinó la prevalencia general y por comunidad de hidatidosis humana con un intervalo de confianza (IC) al 95\%. La prueba de Chi Cuadrado se usó para evaluar la asociación de los resultados de las pruebas diagnósticas con la edad, género y lugar de residencia. Además, se estableció la relación de quiste hidatídico hepático / pulmonar.

\section{Resultados}

El tamaño de la población en las comunidades participantes fue de 1973 pobladores (6.9\% menores de 5 años), de los cuales, 949 decidieron participar en el estudio (51.7\%) y fueron evaluadas mediante el examen ecográfico. Las radiografías de tórax solo se tomaron a 829 personas $(45.2 \%)$.

Los exámenes revelaron 45 (4.7\%) y 9 (1.1\%) casos de quistes hidatídicos hepáticos y pulmonares, respectivamente. Dentro de este grupo, dos personas presentaron quistes en hígado y pulmón. La prevalencia general de hidatidosis fue 5.5\% (52/949) con un IC de 4.1 a $7.1 \%$. No obstante, ningún paciente presentó sintomatología clínica durante las pruebas. Se observó una asociación estadística significativa $(\mathrm{p}<0.05)$ entre la variable edad y los resultados del examen ecográfico; donde las personas mayores de 40 años presentaron prevalencias más altas de quistes hidatídicos en comparación con personas más jóvenes (Cuadro 1). 
Cuadro 1. Quistes hidatídicos detectados por ecografía y radiografía de tórax en personas de nueve comunidades del distrito de Yanahuanca, Pasco (2004)

\begin{tabular}{|c|c|c|c|c|}
\hline \multirow{2}{*}{$\begin{array}{l}\text { Edad } \\
\text { (años) }\end{array}$} & \multicolumn{2}{|c|}{ Ecografía $^{1}$} & \multicolumn{2}{|c|}{ Radiografía } \\
\hline & $\begin{array}{c}\text { Examinados } \\
\text { (n) }\end{array}$ & $\begin{array}{c}\text { Positivos } \\
\text { (\%) }\end{array}$ & $\begin{array}{l}\text { Examinados } \\
\text { (n) }\end{array}$ & $\begin{array}{c}\text { Positivos } \\
\text { (\%) }\end{array}$ \\
\hline 5 a 9 & 159 & 1.9 & 148 & 0.7 \\
\hline 10 a 19 & 264 & 4.2 & 237 & 1.3 \\
\hline 20 a 39 & 274 & 2.9 & 231 & 1.7 \\
\hline 40 a 59 & 147 & 8.2 & 120 & 0 \\
\hline$\geq 60$ & 105 & 10.5 & 93 & 1.1 \\
\hline Total & 949 & 4.7 & 829 & 1.1 \\
\hline
\end{tabular}

En los quistes hidatídicos de localización hepática se detectó 25 (55.6\%) casos positivos a quiste tipo CE5, seguido por el tipo CE1 y CE2 con 8 (17.8\%) y 4 (8.9\%) casos, respectivamente. Se detectaron 40 casos con quistes únicos y cinco con dos quistes, haciendo un total de 50 quistes hepáticos. Por otro lado, el número de quistes pulmonares fue de 10 (5 en pulmón izquierdo, 3 en el derecho y 1 en ambos). La relación hígado / pulmón fue 5:1.

\section{Discusión}

La prevalencia de hidatidosis humana encontrada en las nueve comunidades del distrito de Yanahuanca (5.5\%) constituye un serio problema de salud pública. Se han descrito prevalencias similares en zonas rurales de la Sierra Central, con prevalencias que van desde 4.9 a 9.3\% (Moro et al., 1999, 2005). Asimismo, la prevalencia hallada en este estudio es una de las más altas reportadas en Latinoamérica. Así tenemos que países de la región considerados endémicos han reportado prevalencias de 1.6 y 3.6\% (Carmona et al., 1998; Cohen et al., 1998) en Uruguay, y 1.1 y 0.2\% (Larrieu et al., 2002; Zanini, 2002) en Argentina.
La alta prevalencia observada en el presente estudio, a diferencia de otros países endémicos, puede deberse a varios factores. Uno de los más importantes es, probablemente, la ausencia de un programa integral de control de hidatidosis, principalmente en los departamentos de Pasco, Junín, Huancavelica, Puno y algunas provincias de Lima, tal como lo ha mencionado Salvatella (2005) en un informe para el Proyecto sub-regional de control y vigilancia de Hidatidosis en el Cono Sur. Esta situación no es nueva, ya que Moro et al. (1997) sugirieron que el incremento de la prevalencia de hidatidosis humana en la SAIS Túpac Amaru fue consecuencia del cese prematuro del Programa Piloto de Control en 1980. Por otro lado, países como Argentina, Chile y Uruguay mantienen sus medidas de control contra esta parasitosis, basadas en la desparasitación de perros con praziquantel cada 45 días, así como una legislación para regular la población canina, control de la faena en mataderos oficiales y educación sanitaria. Todas estas actividades son consideradas exitosas para la disminución de la prevalencia e incidencia de hidatidosis humana y animal (Jiménez et al., 2004; Larrieu et al., 2004; Salvatella, 2005).

Los casos positivos del presente estudio podrían explicarse por la convivencia y 
estrecho contacto del poblador andino con el perro (Carmona et al., 1998; Cohen et al., 1998; Campos et al., 2000). Esta convivencia está vinculada al rol que tienen los perros en las zonas rurales altoandinas, pues ellos intervienen en las faenas del cuidado y pastoreo del ganado ovino (Núñez et al., 2003). Esto, sumado a las deficientes condiciones higiénico-sanitarias, los hábitos y costumbres propios del poblador andino, y los bajos niveles socioeconómicos y culturales de la población, permitirían el mantenimiento de niveles de transmisión del E. granulosus del perro al hombre (Leguía, 1999; Chuquisana et al., 2000).

Los grupos de mayor edad presentaron una mayor ocurrencia de quistes al examen ecográfico, resultados compatibles con otros estudios (Carmona et al., 1998; Cohen et al., 1998). Este mismo comportamiento se ha descrito en ovinos iguales o mayores de 6 años de edad, los cuales presentaron una mayor prevalencia (96\%) en comparación con los más jóvenes. Se ha reportado que los animales menores de un año de edad tienen una prevalencia de $40 \%$, la cual se incrementa conforme avanza la edad del animal (Dueger y Gilman, 2001). La mayor prevalencia hallada en el grupo de personas de 40 años podría sugerir que la ingestión de los huevos de E. granulosus ocurriría en etapas tempranas de la vida seguido por un lento crecimiento del quiste hidatídico. Esto reflejaría que tal ingestión se produciría en cantidad y frecuencia aparentemente bajas, que no serían suficientes para estimular una adecuada inmunidad (Cabrera et al., 1995) permitiendo el establecimiento y desarrollo del metacéstode.

La relación entre quistes hidatídicos hepáticos y pulmonares fue 5:1, mostrando una aparente sensibilidad del hígado de presentar mayor intensidad de infección en comparación a los pulmones. Se ha descrito que la susceptibilidad hepática podrá estar vinculada al efecto de «filtro» venoso y la retención de los estadios iniciales del parásito a nivel de este órgano (Larrieu et al., 2000). Sin embargo, en Argentina, Chile y Uruguay, dicha relación varía entre 3:1 a 13:1. Esto igualmente contrasta con los hallazgos realizados en la SAIS Túpac Amaru y en la comunidad de Vichaycocha (Sierra de Lima), donde se reportan relaciones de 1.4:1 y 1.75:1, respectivamente (Moro et al., 1999). Los autores de esos estudios propusieron como posibles causas de una mayor frecuencia de quistes pulmonares el efecto de la altura y el incremento consecuente del volumen de sangre y la dilatación de los capilares pulmonares. Adicionalmente, existe la hipótesis que la diferencia en la tasa de infección del órgano se deba a un tropismo pulmonar asociado a la presencia de una cepa local de $E$. granulosus (Moro et al., 1999).

El hallazgo de 25 (50\%) casos de quistes hidatídicos hepáticos con parcial o completa calcificación es contradictorio al obtenido por Moro et al. (2005) en Canchayllo (Junín), donde reportaron menos del 10\% de lesiones hepáticas calcificadas. Esta diferencia podría deberse a la presencia de una cepa más benigna, la historia natural de degeneración y calcificación de quistes, a un desarrollo de la inmunidad individual de los pobladores de la zona de Yanahuanca, o a tratamientos antiparasitarios recibidos contra otros parásitos. Sin embargo, los resultados obtenidos en lo que respecta a quistes calcificados no fueron concluyentes.

\section{ConClusiones}

- La prevalencia de hidatidosis humana en nueve comunidades rurales del distrito de Yanahuanca, Pasco, fue de 5.5\% (52/ 949) con un intervalo de confianza al $95 \%$ de 4.1 a $7.1 \%$, utilizando como métodos de diagnóstico la ecografía y radiografía.

- Hubo asociación estadística entre la edad y los pobladores positivos a quistes hidatídicos en el examen ecográfico, donde las personas de 40 años a más presentaron mayor prevalencia.

- Se encontró una relación de quistes hidatídicos hepáticos: pulmonares de 5:1. 


\section{LiTERATURA CitadA}

1. Aliaga F, Oberg C. 2000. Epidemiología de la hidatidosis humana en la IX Región de la Araucanía, Chile. 1991-1998. Bol Chil Parasitol 55(3-4): 54-58.

2. Beggs I. 1985. The radiology of hydatid disease. Am J Roentgenol 145: 639-648.

3. Cabrera P, Haran G, Benavides $U$, Valledor S, Perera G Lloyd S, et al. 1995. Transmission dynamics of Echinococcus granulosus, Taenia hydatigena and Taenia ovis in sheep in Uruguay. Int J Parasitol 25: 807-813.

4. Campos A, López G, Andrés A. 2000. Risk factors for Echinococcus granulosus infection: a case-control study. Am J Trop Med Hyg 62: 329-334.

5. Carmona C, Perdomo A, Carbo A, Alvarez C, Monti $J$, Grauert $R$, et al. 1998. Risk factors associated with human cystic Echinococcosis in Florida, Uruguay: Results of a mass screening study using ultrasound and serology. Am J Trop Med Hyg 58: 599-605.

6. Chuquisana J, Chávez A, Casas E. 2000. Determinación de Echinococcus granulosus en perros del cono norte de Lima. Rev Inv Vet, Perú 11(2): 24-29.

7. Cohen H, Paolillo E, Bonifacio R, Botta B, Parada L, Cabrera P, et al. 1998. Human cystic Echinococcosis in a Uruguayan community: a sonographic, serologic, and epidemiologic study. Am J Trop Med Hyg 59: 620-627.

8. Dueger E, Gilman R. 2001. Prevalence, intensity, and fertility of ovine cystic echinococcosis in the central Peruvian Andes. T Roy Soc Trop Med H 95: 379-383.

9. Eckert J, Deplazes P. 2004. Biological, epidemiological and clinical aspects of Echinococcosis a zoonosis of increasing concern. Clin Microbiol Rev 17(1): 107-135.

10. Jiménez S, Pérez A, Juste R, Quiñones C. 2004. Diecisiete años de programa control de la hidatidosis en la Rioja: resultados y valoración económica. Bol Epidemiol 196: 1415-1418.
11. Larrieu E, Belloto A, Arambulo P, Tamayo H. 2004. Echinococcosis quística: epidemiología y control en América del Sur. Parasitol Latinoam 59: 82-89.

12. Larrieu E, Del Carpio M, Salvitti J, Sustersic J, Panomarenko $H$, Mercapide C, et al. 2002. Diagnóstico y tratamiento de la hidatidosis en población escolar: informe preliminar. Arch Argent Pediatr 100(6): 448-455.

13. Larrieu E, Frider B, Del Carpio M, Salvitti J, Mercapide C, Pereyra R, et al. 2000. Portadores asintomáticos de hidatidosis: epidemiología, diagnóstico y tratamiento. Rev Panam Salud Pub 8(4): 250-256.

14. Leguía G. 1999. Enfermedades parasitarias y atlas parasitológico de camélidos sudamericanos. Lima: Ed De Mar. 190 p.

15. Moro P, Bonifacio N, Gilman $R$, Lopera L, Silva B, Takumoto $R$, et al. 1999. Field diagnosis of Echinococcus granulosus infection among intermediate and definitive hosts in an endemic focus of human cystic echinoccosis. T Roy Soc Trop Med H 93: 611-615.

16. Moro P, García H, Gonzáles A, Bonilla J, Verástegui M, Gilman $R$. 2005. Screening for cystic echinococcosis in an endemic region of Peru using portable ultrasonography and the enzyme-linked immunoelectro-transfer blot (EITB) assay. Parasitol Res 96: 1350-1356.

17. Moro PL, Gilman RH, Verastegui M, Bern C, Silva B, Bonilla JJ. 1999. Human hidatidosis in the Central Andes of Peru: evolution of the disease over 3 years. Clin Infect Dis 29: 807-812.

18. Moro P, McDonald J, Gilman J, Silva $H$, Verástegui M, Malqui $M$, et al. 1997. Epidemiology of Echinococcus granulosus infection in the central Andes of Peru. B World Health Organ 75: 553-561.

19. Náquira C, Bullón F, Balvín G Reyes $N$. 1989. Epidemiología de la hidatidosis en el Perú. En: Anales del Seminario Nacional de Hidatidosis y Enfermedades de Transmisión Alimentaria. Lima: MINSA. p122-137. 
20. Núñez E, Calero D, Estares L, Morales A. 2003. Prevalencia y factores de riesgo de hidatidosis en población general del distrito de Ninacaca-Pasco, Perú 2001. An Fac Med 64(1): 34-42.

21. Salvatella R. 2005. II Reunión del Proyecto Subregional Cono Sur de control y vigilancia de la Hidatidosis: Argentina, Brasil, Chile y Uruguay. Montevideo: OPS. 37 p.
22. WHO Informal Working Group. 2003. International classification of ultrasound images in cystic echinococcosis for application in clinical and field epidemiological settings. Acta Tropica 85: 253-261.

23. Zanini F. 2002. El programa de control de la hidatidosis de Tierra del Fuego. En: Situación de la hidatidosis en Argentina. Argentina: Ed. Martín. 244 p. 